\title{
A Conceptual Framework for the Use of Minimum Redundancy Linear Arrays and Flexible Arrays in Future Smartphones
}

\author{
Ashish Patwari (iD) and G. Ramachandra Reddy $(\mathbb{D}$ \\ School of Electronics Engineering, VIT University, Vellore, India \\ Correspondence should be addressed to Ashish Patwari; ashishpvit@gmail.com
}

Received 16 May 2018; Accepted 6 August 2018; Published 18 September 2018

Academic Editor: Stefania Bonafoni

Copyright (c) 2018 Ashish Patwari and G. Ramachandra Reddy. This is an open access article distributed under the Creative Commons Attribution License, which permits unrestricted use, distribution, and reproduction in any medium, provided the original work is properly cited.

\begin{abstract}
This work applies existing array processing principles to devise a new area of application. The properties of minimum redundancy linear arrays (MRLAs) and flexible arrays are studied, keeping in mind the possibility of using them in flexible 5G smartphones of the future. Millimeter frequencies for $5 \mathrm{G}$ communications enabled the use of a decent number of array elements, even at the user equipment (UE). MRLAs possess attractive properties among linear sparse arrays and flexible conformal arrays (flexible arrays) operate satisfactorily even when the surface they are built into changes shape. To the best of our knowledge, MRLAs were not applied to smartphones previously. In this work, a 16-element uniform linear array (ULA) and a 7-element MRLA (with the same aperture) are considered for simulations. Array factors of both the arrays in flat and bent positions have been computed using MATLAB. The effect of phase compensation and bending radii on the array pattern were verified. That phase compensation using the projection method (PM) restores the array pattern even for a bent MRLA is a major finding. Possible array processing modes have been suggested for a $5 \mathrm{G}$ smartphone in which the array could be made to operate in any of the four configurations: a flat ULA, a bent ULA, a flat MRLA, and a bent MRLA.
\end{abstract}

\section{Introduction}

Minimum redundancy linear arrays (MRLAs) or minimum redundancy arrays (MRAs) have numerous useful properties and had been primarily studied in the past in relation to radio astronomy $[1,2]$. They belong to the class of linear sparse arrays, a subset of nonuniform linear arrays, and provide the largest aperture (barring minimum hole arrays) for a given number of elements or, conversely, use a minimum number of elements to realize a given aperture [3]. Early evidence on the use of MRAs in digital communications can be found in [4-6]. In the present decade, MRAs have returned into the limelight following the introduction of other types of sparse arrays (such as coprime and nested arrays). It has been proved recently that MRAs are less susceptible to the effects of mutual coupling in comparison to coprime and nested arrays [7]. It was also proved that the mean square error (MSE) and the Cramer-Rao bound (CRB) were the least for MRAs $[8,9]$. Conformal arrays have a long history and have been studied extensively. These arrays lie on or are integrated into the surface of objects such as airborne vehicles, satellites, buildings, or any other structure that has some curvature (i.e., the surface is not necessarily flat) [10].

Conformal arrays for millimeter wave frequencies around $60 \mathrm{GHz}$ have been prototyped in [11,12]. Authors in [11] demonstrated a conformal array bent around a cylindrical surface, much similar to the human wrist (for applications in future mobile devices, wrist watches, etc.), and analyzed the effect of the bending radius on the array pattern. A beam switching mechanism for a convex structure bent around a cylinder, consisting of three antenna arrays was demonstrated in [12], where each antenna array (selected using a single-pole three-throw switch) could orient the main beam in a different direction. An eight-element series-fed microstrip antenna array conformal to a cylindrical surface operating at $35 \mathrm{GHz}$ was designed in [13]. Sidelobes were kept within the desirable limits following a Taylor pattern for element amplitudes. In general, conformal arrays are bent 
around rigid (fixed) surfaces. However, some applications require the array to operate on surfaces that change their shape over time.

The SELFLEX array (flexible microstrip array) designed and prototyped in [14] has the ability to automatically provide the necessary phase compensation needed to recover the array pattern by sensing the amount of deformation undergone by the conformal surface using an inbuilt resistive sensor. This self-adapting ability is extremely useful for surfaces that change shape (curvature) with time. A similar flexible phased array demonstrated in [15] does not even need prior information on the possible shape or curvature that the conformal array can take and uses the instantaneous relative locations of the elements to provide phase compensation in real-time.

Phase compensation using the projection method (PM) depends only on the strain sensor's data for arrays steered towards the broadside but becomes an analytical function of the array deformation and the desired main lobe direction in the case of arrays steered towards other directions (beamtilted arrays). An analytical expression must be evaluated each time that either the deformation shape or the steering angle changes. Authors in [16] simplified the PM technique by formulating the required phase compensation as a sum of two terms-one to compensate the array deformation and the other to steer the main beam towards the desired direction thereby avoiding the need for evaluating the analytical expressions.

Design of antenna arrays for 5G UEs is being studied extensively $[17,18]$. Microstrip patch antennas are generally used for mobile handsets, but metallic frame antennas have received attention recently $[19,20]$. A slotted waveguide antenna array for $5 \mathrm{G}$ indoor applications has been demonstrated in [21]. This is different from the microstrip or substrate-integrated-waveguide (SIW) approaches presently in vogue for antenna design.

To the best of our knowledge, this is the first time that MRAs and their combination with flexible linear arrays are being studied in the context of smartphones. This work stitches together unconnected areas within the broad domain of array processing and comes out with a new application area. As foldable and, eventually, fully flexible handsets are being designed for future smartphones, the applicability of flexible linear arrays deserves some attention.

Our motive was to see whether MRAs and flexible arrays could be combined to fit into the framework of a $5 \mathrm{G}$ smartphone. Off-the-shelf MRA configurations were used for simulations instead of proposing any new algorithms for their synthesis. Array factors of MRAs were compared with those of ULAs that either use the same number of elements or provide the same aperture. Similarly, the role of phase compensation in restoring the pattern of a ULA bent into a semicircular arc was verified. It was also experimented whether phase compensation applied to a bent MRA could recover its pattern. The results were encouraging. Finally, various array processing modes in which the $5 \mathrm{G}$ smartphone could operate were projected. Because the emphasis was to build the conceptual framework around the proposed idea, we did not focus on the aspects such as antenna design, prototyping, choice of materials, algorithms for directionof-arrival (DOA) estimation and beamforming.

The rest of the paper is organized as follows. Section 2 gives an overview of MRAs and the array model for flexible arrays considered in this work. Section 3 discusses the methodology used for simulations. Section 4 describes the numerical simulation results obtained in MATLAB and contains a subsection that brings together MRAs and flexible arrays for a possible application in 5G mobile handsets. Section 5 proposes various predicted array processing modes of a 5G smartphone. Section 6 contains a discussion on the merits of MRAs, their suitability for 5G systems, and provides future directions. Section 7 concludes the paper.

\section{MRAs, Flexible Arrays, and the Array Model}

This section presents the background information on MRAs and describes the array model considered for flexible conformal arrays.

2.1. Minimum Redundancy Arrays. MRAs are synthesized from a full antenna array by eliminating selected antennas while preserving all possible antenna separations [22]. A regular or full array has many antenna element combinations that generate a given spatial lag (e.g., considering the numbers from 0 to 9 , a spatial lag of 3 can be obtained using any of the antenna pairs $\{9,6\},\{8,5\},\{7,4\},\{6,3\},\{5,2\}$, $\{4,1\}$, and $\{3,0\}$. Similarly, any pair among $\{9,2\},\{8,1\}$, and $\{7,0\}$ can provide a spatial lag of 7$)$. MRAs are synthesized by minimizing this redundancy through the careful removal of select antenna elements, such that the antenna elements thus retained can generate all spacings between zero and a specified maximum number. MRAs, like other sparse arrays, can identify more sources than the number of sensors. However, their merit comes from the fact that they form the largest filled coarray and provide the largest aperture among nested, coprime, and supernested arrays for a given number of elements, thereby providing the best resolution.

Let $N_{a}$ denote the maximum required aperture in units of the interelement spacing $d$. The total aperture distance is given by $N_{a} d$. There might exist several MRA configurations which provide the needed aperture. This means that there can exist several combinations of numbers that can generate all the differences between 0 and $N_{a}$. For instance, the numbers $\{0,1,4,6\}$ and $\{0,2,5,6\}$ can generate all the differences between 0 and 6 . Each of these configurations might have a different array response pattern (in terms of beamwidth and sidelobe levels).

The array patterns are computed using the following formula inspired from [23]. Individual elements are assumed to be identical and isotropic.

$$
A F(\phi)=\frac{1}{A F_{\max }} \sum_{n=1}^{N} w_{n} e^{j(n-1) k d \cos \phi},
$$

where $k=2 \pi / \lambda$ is the wavenumber, $d=0.5 \lambda, w_{n}=$ amplitude weight of element $n, w_{n}$ is either 0 (inactive element) or 
1 (active element), $N=$ number of elements in the array, $\phi=$ azimuth angle, $0^{\circ} \leq \phi \leq 180^{\circ}$, and $A F_{\max }=$ maximum value of array pattern, given by

$$
A F_{\max }=\sum_{n} w_{n}
$$

Another notation given by $\{.(a+1) \cdot(b+1) \cdot(c+1) \cdot(d+1)$. is widely used to represent MRAs, where the dot (.) represents a sensor's presence, followed by $a$ voids, which are followed by the second sensor and $b$ voids, and so on. The number of dots added to the sum of the numbers $a, b, c, d$ gives the value equal to the aperture size plus one $\left(N_{a}+1\right)$. Sometimes the notation $\{(a+1)(b+1)(c+1)(d+1)\}$, with the dots removed, is used for simplicity [24].

2.2. Flexible Arrays Bent into Semicircular Arc. Consider a ULA of $N$-elements along the $x$-axis with an interelement spacing of $d=0.5 \lambda$. It is bent into a semicircular arc in the $x y$-plane. The semicircle has a radius of $r$ and is centered at $(r, 0)$ along the $x$-axis. The arrangement ensures that the first and last elements lie on the $x$-axis at $(0,0)$ and $(2 r, 0)$, respectively, even after the bending. The elements remain uniformly spaced even after bending along the semicircular arc, but the new interelement spacing is

$$
d_{b}=\frac{\pi r}{N-1}
$$

The array factor for such a bent or conformal array is given in [10] and repeated here for convenience

$$
A F=\sum_{n=1}^{N} w_{n} e^{j k\left(x_{n} \sin \theta \cos \phi+y_{n} \sin \theta \sin \phi+z_{n} \cos \theta\right)},
$$

where $k, w_{n}$ are as defined in (1). The array is assumed to be beam-steered towards the broadside direction (i.e., $90^{\circ}$ azimuth and $0^{\circ}$ elevation).

An important task in evaluating (4) is to determine the positions or locations of the antenna elements along the bent arc, namely, $\left(x_{n}, y_{n}\right)$. The values of $z_{n}$ and the term $z_{n} \cos \theta$ will be zero as there is no displacement of the elements along the $z$-axis. Let $\alpha_{n}$ denote, in degrees, the angle between two lines, the reference being the line joining the center of the semicircle to the $N^{\text {th }}$ element (last element), and the line joining the center of the semicircle to the $n^{\text {th }}$ antenna element being the second.

$$
\alpha_{n}=\frac{(N-n) * 180^{\circ}}{N-1}, n=1 \text { to } N
$$

In the present scenario, the reference line is the positive $x$-axis. It is intuitive that $\alpha_{1}=180^{\circ}$ and $\alpha_{N}=0^{\circ}$. The element positions are given by

$$
\begin{aligned}
& x_{n}=r+r \cos \left(\alpha_{n}\right), \\
& y_{n}=r \sin \left(\alpha_{n}\right) .
\end{aligned}
$$

The array factor computed using (4) will be distorted as the bending of the array introduces phase changes at the elements owing to the displacement in the $y$-direction. The projection method (PM) is a simple, low-cost, and effective technique of pattern recovery that retrieves the radiation pattern of the deformed array. The amount of phase compensation to be applied at each antenna element is obtained using the PM technique and is given by

$$
\Delta_{n}=-\frac{2 \pi y_{n}}{\lambda}=-k y_{n}
$$

The amount of compensation at each element is proportional to its displacement in the $y$-direction. The first and last elements of the bent array do not need any phase compensation as they still lie on the $x$-axis even after bending. The modified or phase compensated array factor is given by

$$
A F_{\text {comp }}=\mathrm{e}^{j \Delta_{n}} \times A F
$$

Three cases mentioned below are considered to determine the radius of the semicircular arc for further evaluation. However, only one of them needs to be selected for the design.

Case 1. The radius is chosen such that the perimeter of the semicircle is equal to the aperture distance of the original ULA. That is $\pi r=(N-1) d=(N-1) \lambda / 2$ and

$$
r=\frac{(N-1) \lambda}{2 \pi}
$$

Case 2. The radius is chosen such that the diameter of the semicircle is equal to the aperture distance of the ULA. This implies $2 r=(N-1) d=(N-1) \lambda / 2$ and

$$
r=\frac{(N-1) \lambda}{4}
$$

Case 3. The radius is arbitrarily fixed at a value.

$$
r=0.3 N \lambda
$$

2.3. The Case of a $5 G$ Smartphone. We consider the coordinate system of the smartphone handset as defined in [25] for describing the placement of the antenna array. The longer dimension of the handset is along the $y$-axis and the smaller along the $x$-axis. The antenna array (ULA) can be placed along the $y$-axis.

2.3.1. Flexible Array Bent in the yz-Plane. If the array (i.e., the handset) is flexed, the displacement would be along the $z$-axis. Figure 1 shows the scenario. The displaced elements are indicated by $s_{n}^{\prime}$, whereas the regular elements are denoted by $s_{n}$. The first and last elements lie on the $y$-axis even after bending the array. 


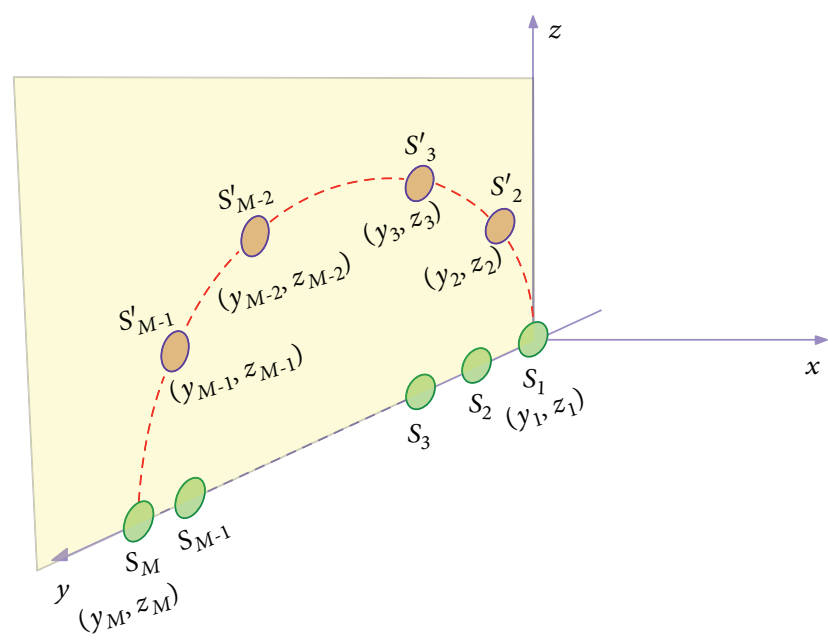

FIgURE 1: Uniform linear array (ULA) along the $y$-axis bent into a semicircular arc in the $y z$-plane.

The semicircle is now centered at $(0, r)$ along the $y$-axis. The array factor in (4) simplifies to

$$
A F_{y z}=\sum_{n=1}^{N} w_{n} e^{j k\left(y_{n} \sin \theta+z_{n} \cos \theta\right)}
$$

because the values of $x_{n}$ and associated terms become zero. Since $\phi=90^{\circ}$ on the $y$-axis, the term $\sin \phi=1$ does not feature in (12). The positive $y$-axis acts as the reference line to determine the angles $\alpha_{n}$ in (5). The element positions, in this case, are given by

$$
\begin{aligned}
& y_{n}=r+r \cos \left(\alpha_{n}\right), \\
& z_{n}=r \sin \left(\alpha_{n}\right) .
\end{aligned}
$$

The amount of phase compensation to be applied to each element is now proportional to the displacement along the $z$-axis and is given by

$$
\delta_{n}=-\frac{2 \pi z_{n}}{\lambda}=-k z_{n}
$$

The phase compensated array factor is given by

$$
A F_{y z, \mathrm{comp}}=e^{j \delta_{n}} \times A F_{y z}
$$

2.3.2. The Wrist-Wrapped Phone. A flexible phone will most likely have a provision to be wrapped around the human wrist. In such a case, the array would still be in the $y z$-plane but bent downwards along the negative $z$-axis. The semicircle is now assumed to be centered at $(0$ $, 1.5 r,-r)$ in the $y z$-plane (obtained heuristically). The first and last elements of the bent array do not lie on the $y$-axis as in the previous scenario. Equations (12), (14), and (15) are still valid whereas the element positions are given by

$$
\begin{aligned}
& y_{n}=1.5 r+r \cos \left(\alpha_{n}\right), \\
& z_{n}=-r+r \sin \left(\alpha_{n}\right) .
\end{aligned}
$$

2.3.3. Beam-Steering the Bent Array: The Modified Phase Compensation Formula. Beam-steering is the process of orienting the main lobe of an array towards a specific direction of interest. A linear array could be beam-steered only in one direction (i.e., either along the azimuth or along the elevation). On the other hand, two-dimensional arrays (such as circular and rectangular arrays) could be beam-steered in both the directions. The azimuth steering angle is denoted by $\phi_{s}$, and the elevation steering angle is denoted by $\theta_{s}$.

The smartphone array which is bent in the $y z$-plane can be beam-steered in the elevation direction. To beam-steer a bent array, the phase compensation provided (using the projection method) should be able to compensate the bending effects as well as achieve beam-steering. As mentioned in [16], the phase compensation formula in this case has two terms: one to account for bending and the other to account for beam-steering. The uncompensated array factor of the bent array is the same as that given in (12). However, the phase compensation formula must be modified as follows

$$
\delta_{n, c s}=-k\left(\mathrm{z}_{n}+y_{n} \sin \theta_{s}\right)=-k z_{n}-k y_{n} \sin \theta_{s} .
$$

The first term indicates the phase shifts to be provided at each array element to compensate the effect of bending. The second term denotes the phase shifts to be applied at each element to steer the main beam towards the desired elevation angle $\theta_{s}$. The basis for (17) is a similar expression given in [26] which was used to compensate and beam-steer an array bent in the $x y$-plane along its azimuth.

The overall compensated and beam-steered array factor is obtained by combining (12) and (17) such that

$$
A F_{y z, C S}=e^{j \delta_{n, c s}} \times A F_{y z} .
$$

\section{Simulation Methodology}

This section describes the methodology followed for simulations. Simulations were done in MATLAB. The ULA size was fixed to 16 elements considering the form factors of the present-day smartphones (6-inch) and an operating frequency of $30 \mathrm{GHz}$. Antenna elements were not customized to any specific type, and therefore, the element patterns do not come into picture in our analysis. Point sources were assumed. The far field pattern depends only on the array factor and can be evaluated mathematically.

3.1. MRA Array Factor Calculation. The first step in computing the array factors of the MRAs is to determine the values $w_{n}$ corresponding to each MRA configuration and use them in (1). A given MRA configuration, which either lists the element positions or is in the form $\{(a+1)(b+1)(c+1)(d+1)\}$ has to be converted into the $w_{n}$ form. For example, the values

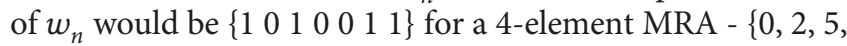


6 that can provide an aperture of $6 d$. The array factor is then computed using (1). Alternatively, one can also compute the array factor using the element positions $x_{n}$ as given below

$$
A F(\phi)=\frac{1}{A F_{\max }} \sum_{n \in M R A} e^{j k x_{n} \cos \phi}
$$

For the 4-element MRA mentioned in the preceding paragraph, the element positions normalized to the wavelength are given by $\{0.0,1.0,2.5,3.0\}$.

3.2. Array Factor for the Flexible Linear Array. The following steps are to be followed to evaluate the array response of the flexible or bent array.

(i) Selecting the radius of the semicircle

(ii) Calculating the angles, $\alpha_{n}$, to determine the element positions

(iii) Computing the uncompensated array pattern

(iv) Finding the amount of phase compensation needed at each element

(v) Evaluating the modified array pattern

The element weights are given by $w_{n}=1 \forall n$ for a bent ULA. Simulation parameters are listed in Table 1.

A total of 77 different MRA configurations can provide the required aperture of $N_{a}=15$. Some of these configurations were tabulated in [27]. We consider a specific MRA configuration whose element positions are given by $\{0,1$, $3,6,10,14,15\}$ for simulations. We refer this MRA as M2 (as it appeared in the second row of the table given in [27] for $\left.N_{a}=15\right)$. The values, $w_{n}$, for $M 2$ are $\left\{\begin{array}{llll}1 & 1 & 0 & 1\end{array}\right.$ $\left.\begin{array}{lllllllllllll}0 & 0 & 1 & 0 & 0 & 0 & 1 & 0 & 0 & 0 & 1 & 1\end{array}\right\}$.

\section{Results}

This section presents the numerical simulation results obtained using MATLAB 2016a.

4.1. MRA Response. The array pattern of the MRA is compared with that of ULAs containing 7 and 16 elements, respectively. This is because the MRA matches a 7-element ULA in the number of elements and resembles a 16element ULA in the aperture provided. It is to be noted that $w_{n}=1 \forall n$ is considered for the ULAs. Figure 2 shows the comparison. The MRA response evaluated using (1) and (19) produced the same result.

It can be observed from Figure 2 that the MRA provides a beamwidth comparable to that of the 16-element ULA, which is much sharper than the beamwidth of the 7element ULA. Since MRAs save on the number of elements, the penalty comes in the form of increased sidelobe levels. This can be overlooked since a 7-element MRA providing the same aperture as a 16 -element ULA, results in a $56.25 \%$ $(((16-7) / 16) \times 100 \%)$ saving in the number of elements and the costs associated thereof (e.g., feed, power consumption, and radio frequency chains). A definite advantage with
Table 1: Simulation Parameters.

\begin{tabular}{lc}
\hline Parameter & Value \\
\hline ULA size & $N=16$ \\
Center frequency $\left(f_{c}\right)$ & $30 \mathrm{GHz}$ \\
Wavelength $(\lambda$ in $\mathrm{m})$ & $0.01 \mathrm{~m}$ \\
Interelement spacing $(d)$ & $d=0.5 \lambda=0.005 \mathrm{~m}$ \\
Maximum aperture obtained & $N_{a}=15$ \\
Total aperture distance & $N_{a} \times d=15 d=0.075 \mathrm{~m}$ \\
& Case $1: r=2.38 \lambda$ \\
Radius of the semicircular arc & Case $2: r=3.75 \lambda$ \\
& Case $3: r=4.8 \lambda$ \\
\hline
\end{tabular}

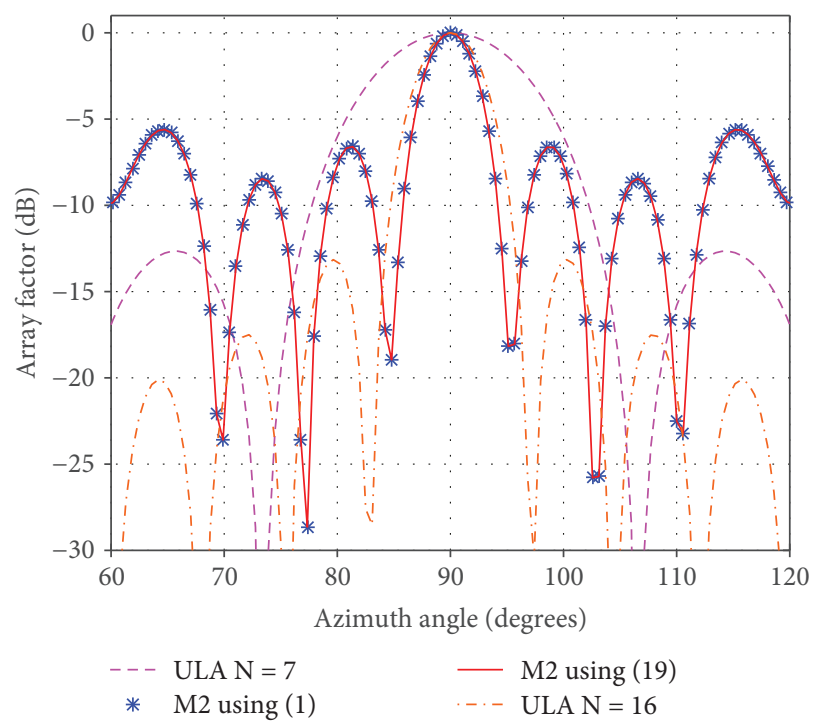

Figure 2: Array patterns of MRA M2, ULAs of 7- and 16-elements.

MRAs is that they provide an acceptable performance using less than half of the total elements in the full array.

4.2. Flexible Linear Array Response. Three different cases are evaluated for the 16-element ULA bent into a semicircle. The radius of the semicircle is fixed using (9), (10), and (11), respectively, for each case. Figure 3 shows the positions of the antenna elements when the array is bent into semicircular arcs of radii $2.38 \lambda, 3.75 \lambda$, and $4.8 \lambda$, respectively, in comparison to the flat ULA case. The response of the bent array with and without phase compensation is calculated using (4) and (8), respectively, and shown in Figure 4. The beamwidth provided by the bent array in these three cases is $8.4^{\circ}, 5.3^{\circ}$, and $4.2^{\circ}$, respectively.

The results depicted in Figure 4 are in accordance with the conventional knowledge pertaining to conformal arrays. Conformal arrays bent around surfaces of larger radii exhibit better response (narrow beamwidths and more gains) than the arrays bent around surfaces of smaller radii $[11,28]$. The smallest radius $2.38 \lambda$ implies that the array undergoes the highest amount of bending (deformation) from its regular position. Larger radii do not strain the array as much as the smaller ones do. For all further simulations, 


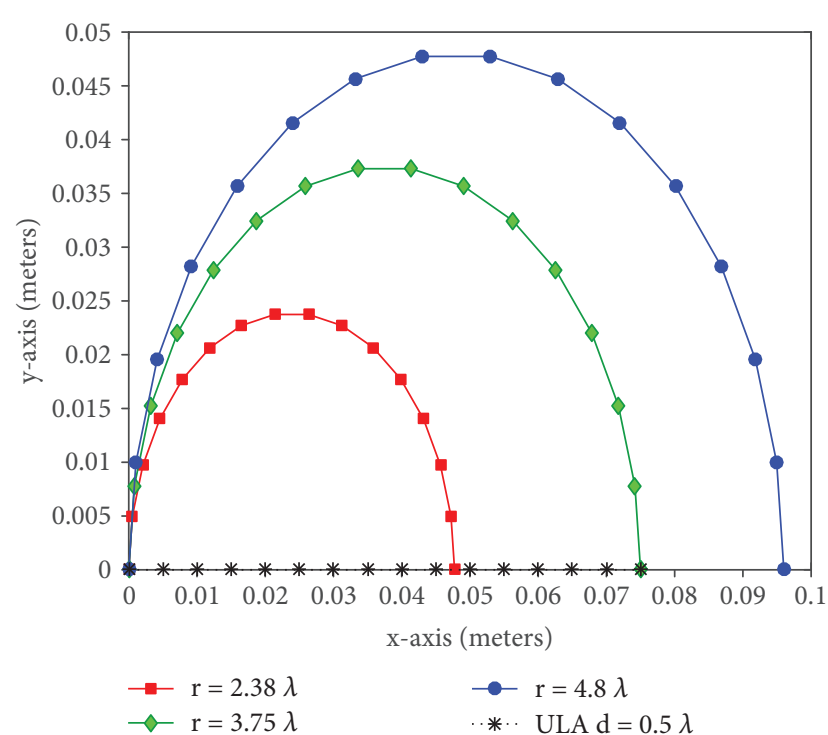

FIGURE 3: Element positions for the ULA when bent into arcs of different radii.

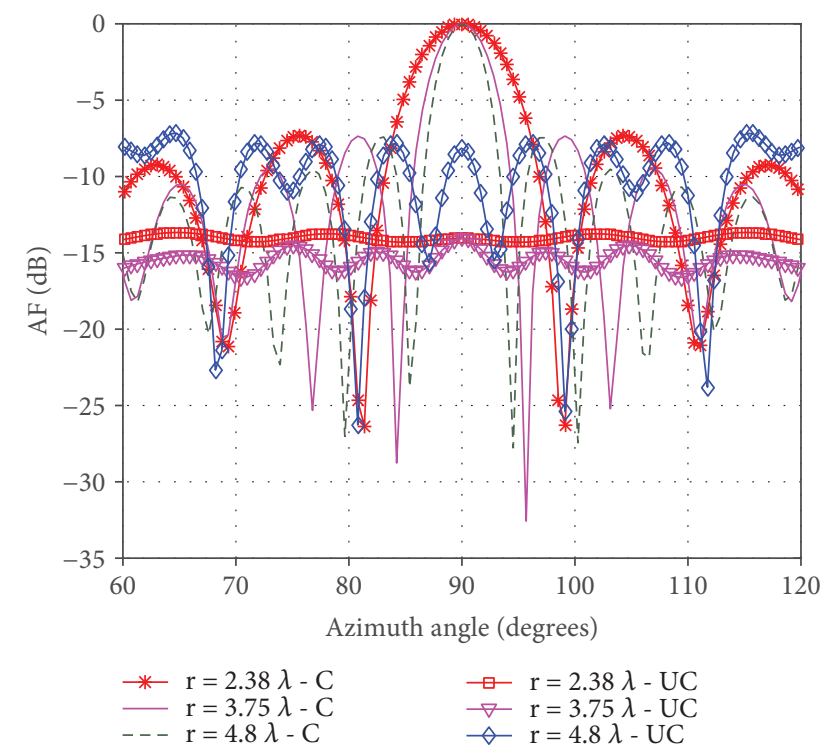

Figure 4: Array factors of the ULA when bent into arcs of different radii (legend entries: C indicates compensated; UC indicates uncompensated).

we fix the radius as $2.38 \lambda$ because the aperture of the bent array should confine to the physical dimensions of the mobile handset when the bent array is brought back to the flat position.

4.3. Joining the Dots: Use of MRAs and Flexible Arrays for the $5 G$ Smartphone. Since a basic understanding of the properties of MRAs and flexible arrays is obtained, we have tried to combine these two aspects in the context of a flexible $5 \mathrm{G}$ smartphone. As described in Section 2, the $y z$-plane (the elevation plane) has to be considered to evaluate the array factor. The array is placed along the $y$-axis.

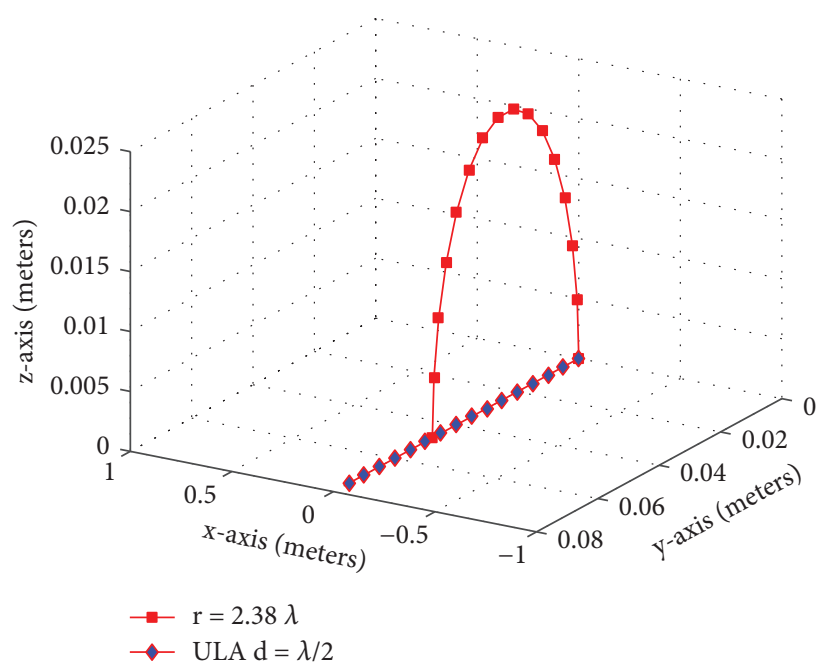

Figure 5: Element positions for the ULA in flat and bent (in the $y z$-plane) positions.

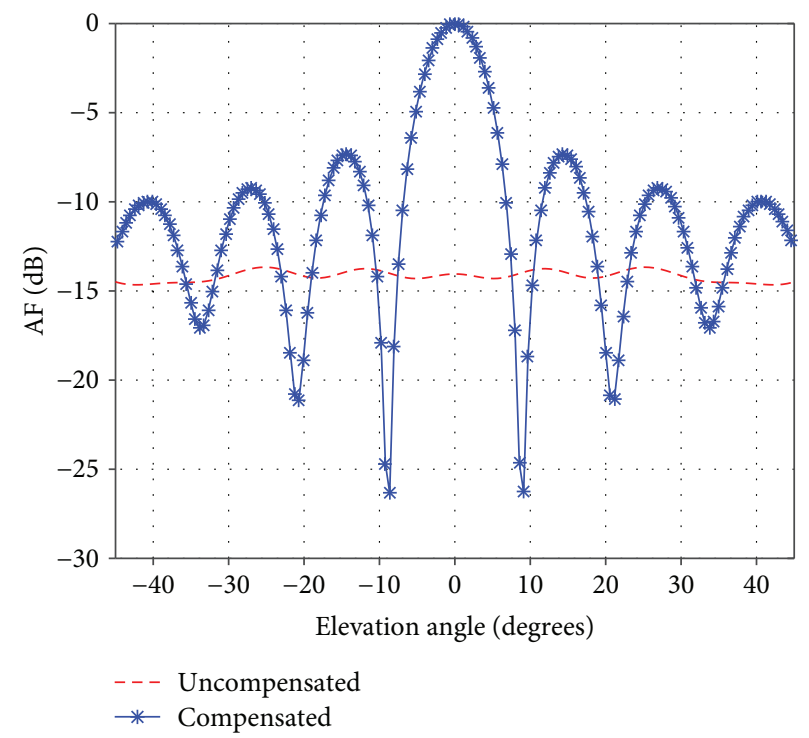

Figure 6: Array factor for the ULA bent in the $y z$-plane with $\mathrm{r}=2.38 \lambda$.

4.3.1. Flexible Array in the yz-Plane: The Bent ULA. The ULA gets displaced to the $y z$-plane when the smartphone is bent. The radius of the semicircular arc is chosen according to (9), and the element positions are calculated using (13). The array factor of the bent array before and after phase compensation is computed using (12) and (15), respectively. Figure 5 shows the element positions, and Figure 6 shows the array factor for the 16-element bent array. It can be seen from Figure 6 that phase compensation restores the array pattern of the ULA.

4.3.2. The Bent MRA: The Minimum Redundancy Flexible Array. An exciting way forward is to see the response of the MRA when it is bent into the semicircular arc along the $y z$ -plane. It is utmost intriguing to find whether phase compensation can restore its pattern. The values of $w_{n}$ corresponding to the MRA M2 are substituted in (12). The radius, element 


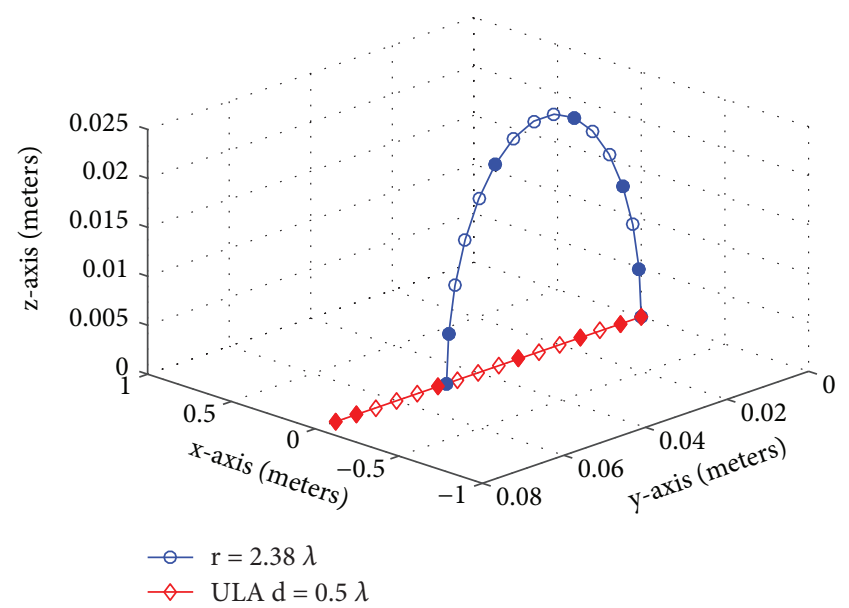

Figure 7: Element positions for the MRA (M2) in flat and bent (in the $y z$-plane) positions.

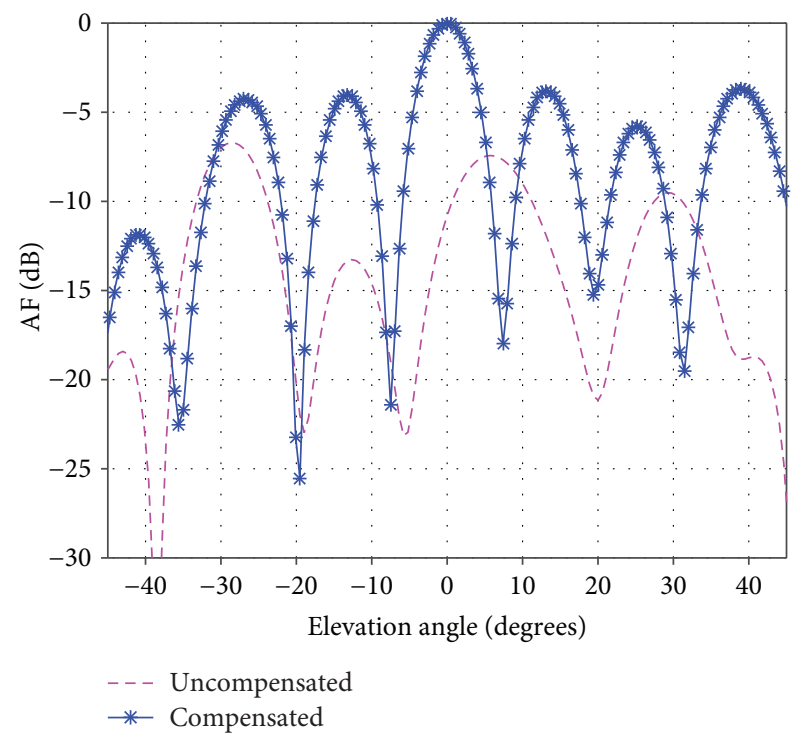

Figure 8: Array pattern of the MRA (M2) bent in the $y z$-plane.

positions, and the array factors are calculated using (9), (13), (12), and (15), respectively. Figure 7 shows the element locations of the flat as well as the bent MRA. The filled shapes in Figure 7 indicate the presence of an element whereas the empty shapes indicate the absence of the same. Figure 8 shows the array response with and without phase compensation.

It can be seen from Figure 8 that like the case of the bent ULA, the radiation pattern of the bent MRA also gets restored upon appropriate phase compensation. To ensure that Figure 8 did not happen by chance, we consider another example. A 6-element MRA capable of providing an aperture of $N_{a}=13$ described in [1] is considered. The MRA is

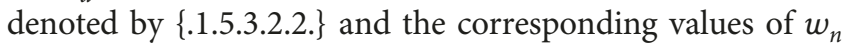

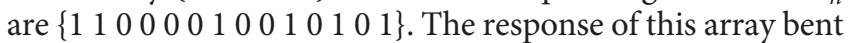
over the semicircular arc with and without phase compensation is shown in Figure 9.

We prove that phase compensation can recover the array patterns of bent MRAs too. This is an interesting result since the phase compensation is applied only to

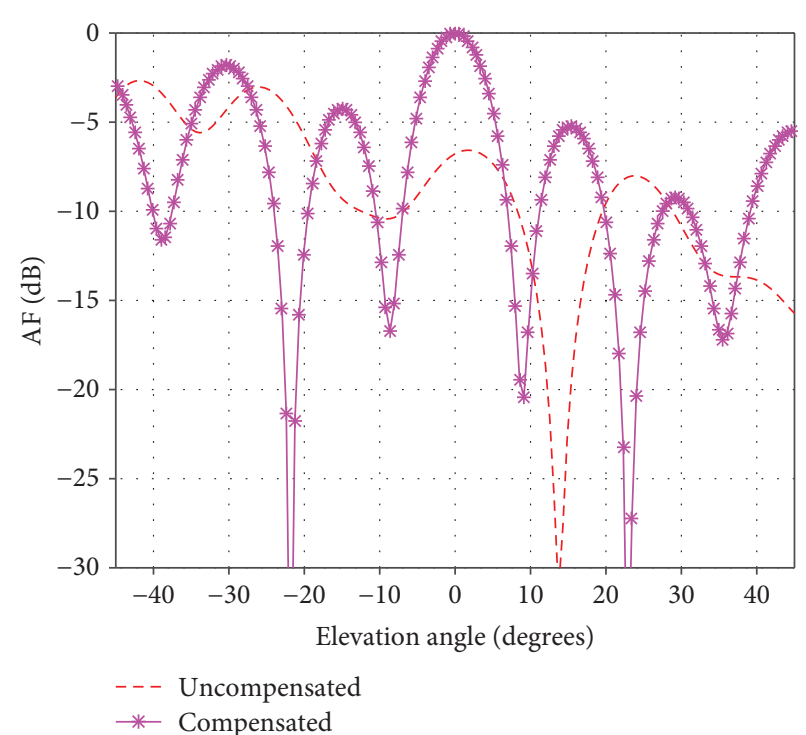

FIgURE 9: Array pattern of the 6-element MRA 15322 with $N_{a}=13$, bent in the $y z$-plane.

TABle 2: Phase compensation at individual elements in bent ULA and bent MRA.

\begin{tabular}{lcccc}
\hline El. no. & $\begin{array}{c}w_{n} \text { for } \\
\text { ULA }\end{array}$ & $\begin{array}{c}\text { Bent ULA phase } \\
\text { compensation }\end{array}$ & $\begin{array}{c}w_{n} \text { for } \\
\text { MRA M2 }\end{array}$ & $\begin{array}{c}\text { Bent MRA phase } \\
\text { compensation }\end{array}$ \\
\hline 1 & 1 & $1.0000+0.0000 \mathrm{i}$ & 1 & $1.0000+0.0000 \mathrm{i}$ \\
2 & 1 & $-0.9997+0.0229 \mathrm{i}$ & 1 & $-0.9997+0.0229 \mathrm{i}$ \\
3 & 1 & $0.9835-0.1811 \mathrm{i}$ & 0 & $0.0000+0.0000 \mathrm{i}$ \\
4 & 1 & $-0.8208+0.5712 \mathrm{i}$ & 1 & $-0.8208+0.5712 \mathrm{i}$ \\
5 & 1 & $0.1510-0.9885 \mathrm{i}$ & 0 & $0.0000+0.0000 \mathrm{i}$ \\
6 & 1 & $0.9114+0.4114 \mathrm{i}$ & 0 & $0.0000+0.0000 \mathrm{i}$ \\
7 & 1 & $-0.1283+0.9917 \mathrm{i}$ & 1 & $-0.1283+0.9917 \mathrm{i}$ \\
8 & 1 & $-0.7037+0.7104 \mathrm{i}$ & 0 & $0.0000+0.0000 \mathrm{i}$ \\
9 & 1 & $-0.7037+0.7104 \mathrm{i}$ & 0 & $0.0000+0.0000 \mathrm{i}$ \\
10 & 1 & $-0.1283+0.9917 \mathrm{i}$ & 0 & $0.0000+0.0000 \mathrm{i}$ \\
11 & 1 & $0.9114+0.4114 \mathrm{i}$ & 1 & $0.9114+0.4114 \mathrm{i}$ \\
12 & 1 & $0.1510-0.9885 \mathrm{i}$ & 0 & $0.0000+0.0000 \mathrm{i}$ \\
13 & 1 & $-0.8208+0.5712 \mathrm{i}$ & 0 & $0.0000+0.0000 \mathrm{i}$ \\
14 & 1 & $0.9835-0.1811 \mathrm{i}$ & 0 & $0.0000+0.0000 \mathrm{i}$ \\
15 & 1 & $-0.9997+0.0229 \mathrm{i}$ & 1 & $-0.9997+0.0229 \mathrm{i}$ \\
16 & 1 & $1.0000+0.0000 \mathrm{i}$ & 1 & $1.0000+0.0000 \mathrm{i}$ \\
\hline
\end{tabular}

select elements which define the MRA. Although the sidelobe levels are high in the compensated array factor, the fact that the main lobe is restored cannot be ignored. We call such an array as the minimum redundancy flexible array (MRFA) or the minimum redundancy conformal array (MRCA). This is a novel method of combining MRAs and flexible arrays.

The phase compensation applied to the elements of the bent ULA and the bent MRA that resulted in Figures 6 and 8 are tabulated in Table 2 . It is seen that the 1 st and the 16 th element do not need any phase compensation. Missing elements that do not feature in the MRA definition are 


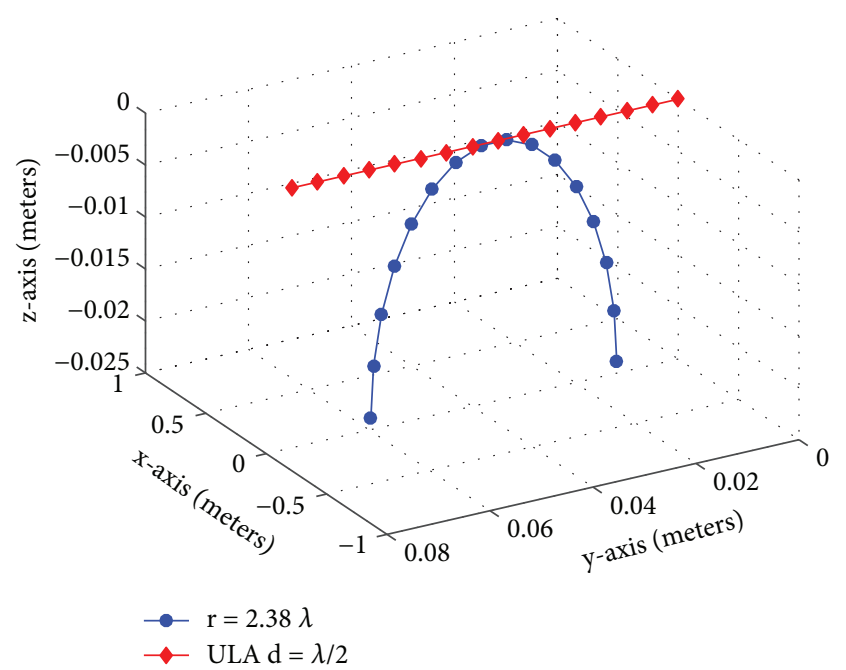

FIGURE 10: Element positions for the ULA in flat and wrist-wrapped positions.

indicated by $w_{n}=0$. Such elements do not (cannot) participate in phase compensation as shown in Table 2.

4.3.3. The Smartphone Wrapped around the Wrist. One of the major motivations behind the design of foldable and/or fully flexible smartphones is the ability to wrap it around the user's wrist. An example design is shown in [29], where the flat phone can be wrapped around the wrist. The radius is fixed using (9), and the element positions are calculated using (16). Figures 10 and 11 show the element positions for the ULA and the MRA wrapped around a semicircular arc resembling the human wrist, respectively.

The response of both the ULA and the MRA, wrapped around the wrist and before and after phase compensation, are identical to the ones shown in Figures 6 and 8, respectively, and are hence not repeated.

4.3.4. Beam-Steering the Bent ULA and the Bent MRA. For the smartphone array bent in the $y z$-plane, the azimuth steering angle was assumed as $\phi_{s}=90^{\circ}$. An elevation steering angle of $\theta_{s}=20^{\circ}$ was considered for simulations. The element positions and the uncompensated array factor were evaluated using (13) and (12), respectively. The compensated and beam-steered array factor was found using (18) and the result is shown in Figure 12. The phase compensation in this case recovers the array pattern and provides beam-steering. Similar results were obtained for the MRA M2 and are shown in Figure 13.

Figure 14, using polar patterns, shows the difference between the results obtained using the original and the modified phase compensation expressions given in (14) and (17) for the bent ULA case. The dotted curve represents the uncompensated response. The dashed curve represents the compensated pattern obtained using (14) and (15). The solid line represents the compensated and beam-steered pattern using (17) and (18). It can be seen that (14) just recovers the array response, whereas (17) recovers the array pattern and additionally provides beam-steering. Figure 15 presents similar results in the case of bent MRA.

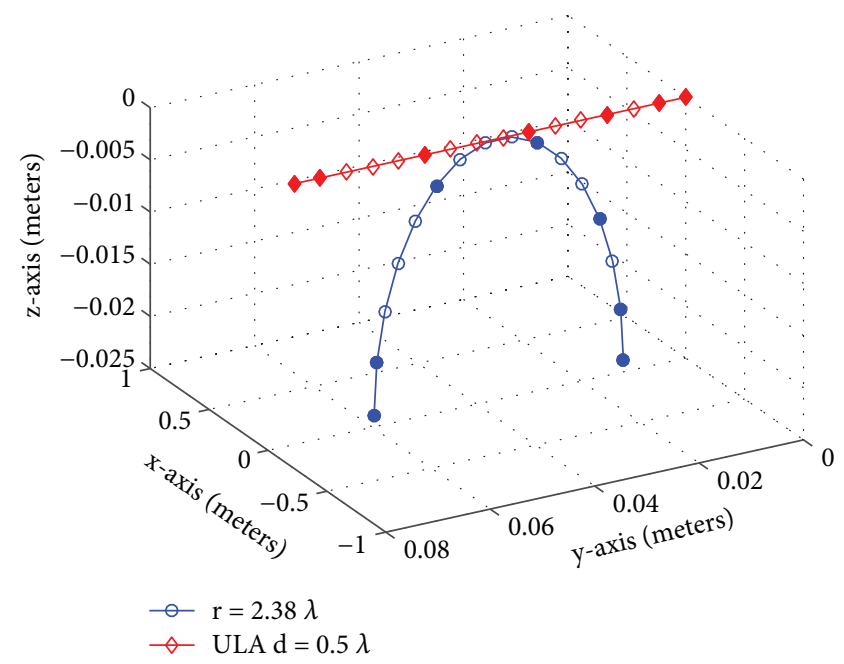

FIgURE 11: Element positions for the MRA in flat and wristwrapped positions.

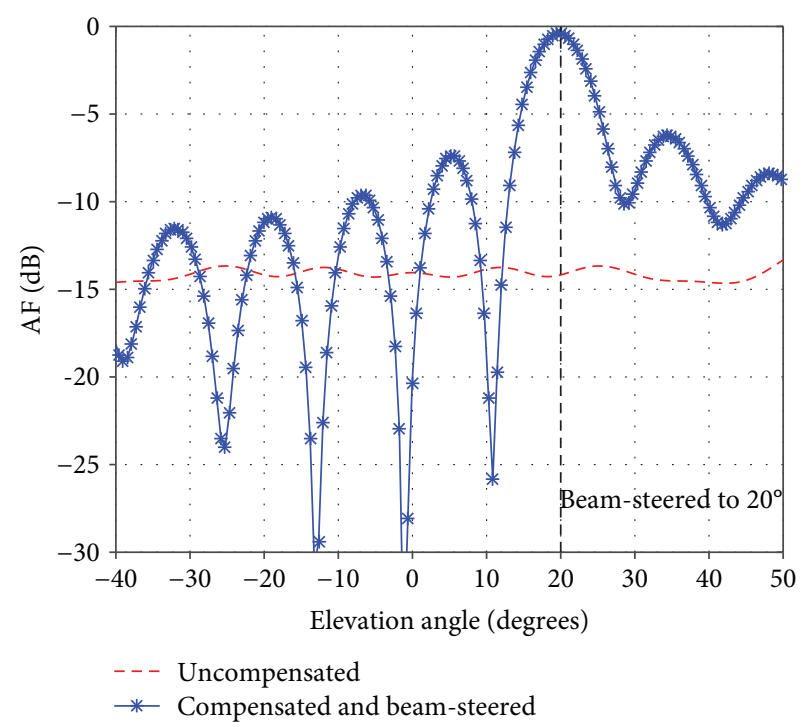

FIgURE 12: Array pattern of the bent ULA after compensation and beam-steering.

Therefore, it can be concluded that the modified phase compensation formula (using the projection method) not only recovers the array pattern but also provides beamsteering and is applicable to both ULAs and MRAs.

\section{The Outcome: Possible Array Processing Modes of the Antenna Array}

Based on the results obtained in the previous section, we define the different modes in which the antenna array could operate. The antenna array can be made to operate in four configurations or states, namely, the flat ULA, the bent ULA, the flat MRA, and the bent MRA. These four configurations can be indicated by $C_{0}, C_{1}, C_{2}$, and $C_{3}$, respectively. 


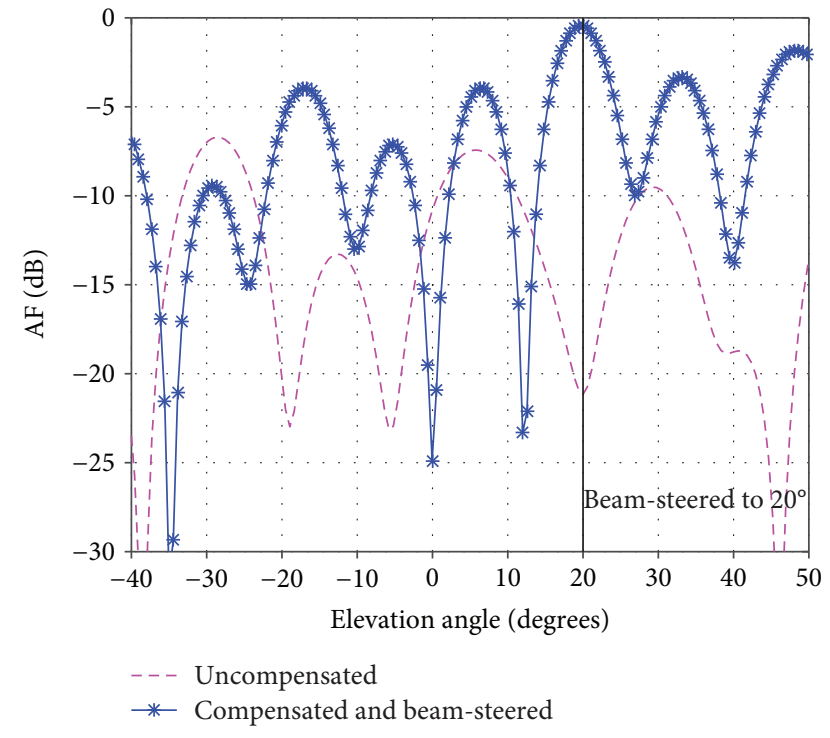

FIGURE 13: Array pattern of the bent MRA after compensation and beam-steering.

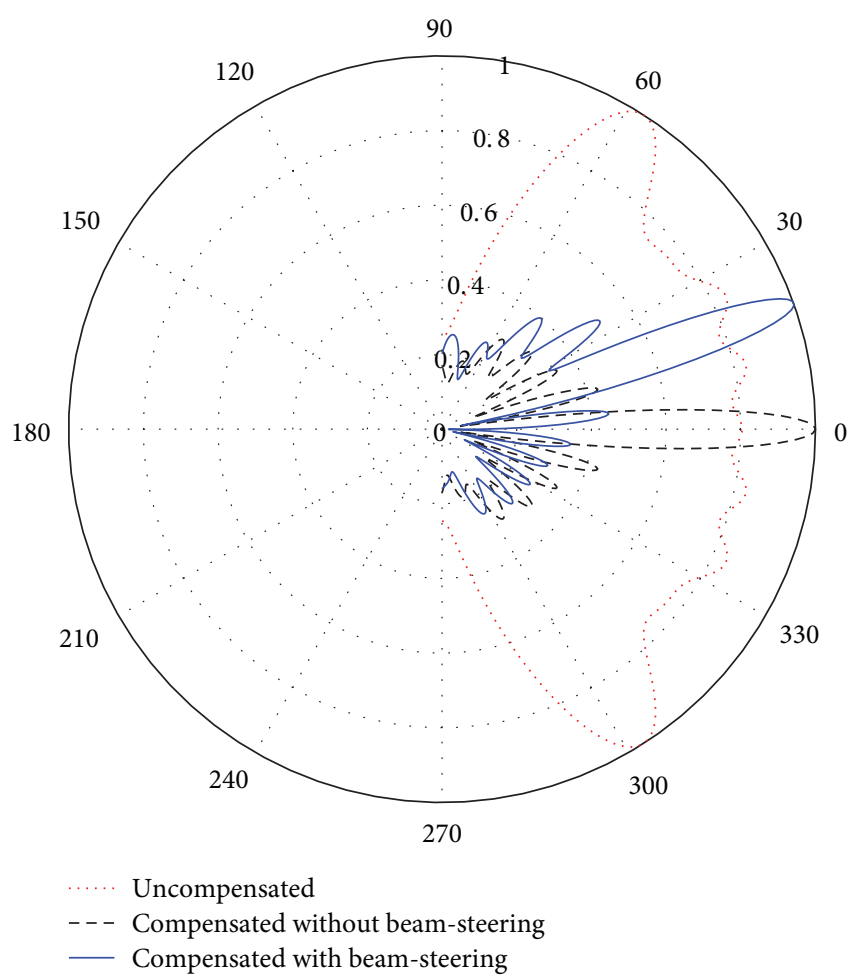

Figure 14: Polar pattern of bent ULA.

5.1. Switching between Array Configurations. The transitions $\left(C_{0} \leftrightarrow C_{1} ; C_{0} \leftrightarrow C_{2} ; C_{2} \leftrightarrow C_{3} ; C_{1} \leftrightarrow C_{3}\right)$ are valid. Figure 16 shows the states and the valid transitions between them. The double arrow indicates a two-way relation between the states.

5.1.1. Realizing the Transitions $C_{0} \leftrightarrow C_{1}$ and $C_{2} \leftrightarrow C_{3}$. The transition $C_{0} \rightarrow C_{1}$ occurs when the phone is bent. A sensing

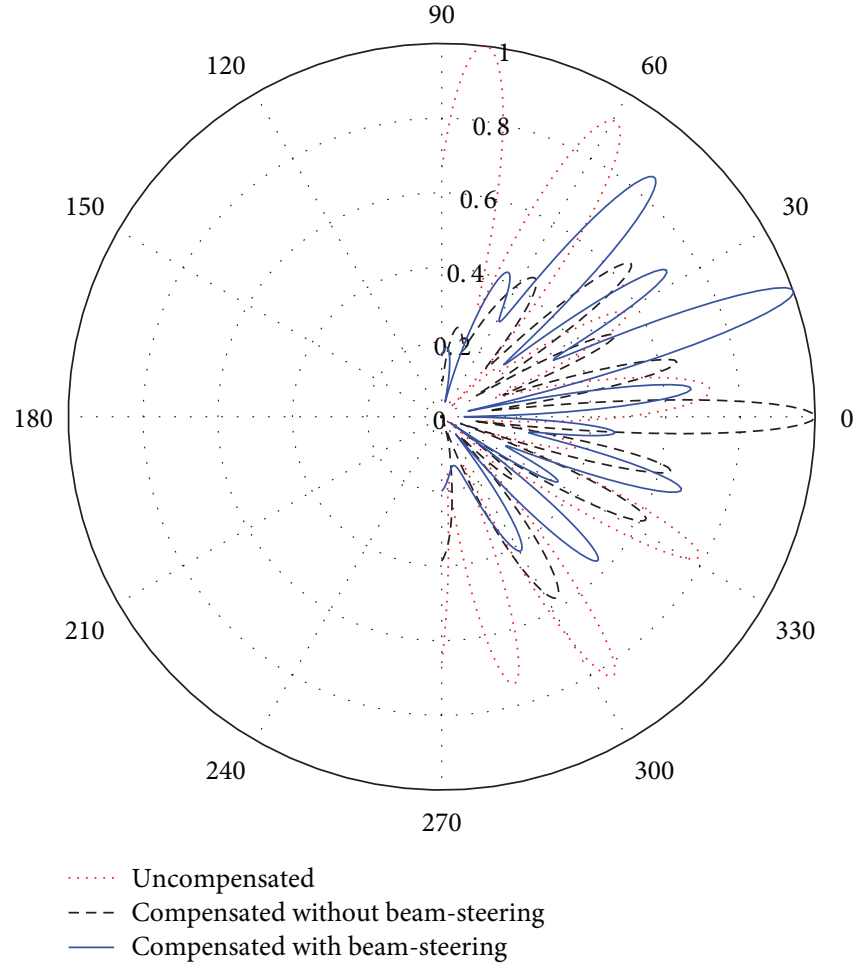

Figure 15: Polar pattern of bent MRA.

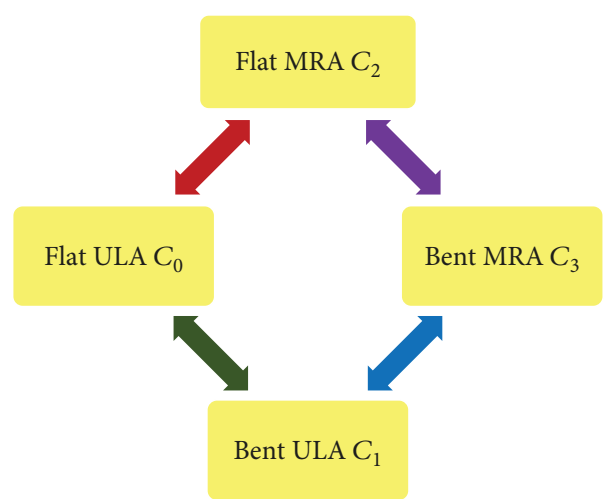

Figure 16: Possible array states and transitions between them.

circuit much similar to the ones described in $[14,15]$ can be made use of for phase compensation. The sensor senses the amount of deformation with reference to the flat case and automatically applies the phase compensation needed to restore the array pattern. In case the main beam is tilted away from the broadside, then the procedure outlined in Subsection 2.3.3 is to be made use of. The same principle applies to the transition $C_{2} \rightarrow C_{3}$. Once the bending force is removed, or the phone is made flat, the sensor no more senses any deformation, the phase compensation process halts, and the transition $C_{1} \rightarrow C_{0}$ or $C_{3} \rightarrow C_{2}$ is realized.

5.1.2. Enabling the Transitions $C_{0} \leftrightarrow C_{2}$ and $C_{1} \leftrightarrow C_{3}$. To realize the transition $C_{0} \rightarrow C_{2}$, a digitally controllable antenna array is required, where the weights of each antenna element can be adjusted. An MRA is realized not by directly 
designing/fabricating it, but by digitally turning off unwanted elements from the full ULA, as per predetermined MRA configurations. This gives a tremendous flexibility in operation as all the elements of the ULA would be still in place, and it is just that they are either activated or deactivated using the weights $w_{n}$. The transition $C_{2} \rightarrow C_{0}$ is quite straightforward and is realized by simply turning on all the elements. Transitions $C_{1} \leftrightarrow C_{3}$ can also be realized using the approach just discussed.

We refer to the full digital multibeam antenna (DMBA) configuration outlined in [30] for this, where each antenna element is tunable in amplitude and phase using digital weights. It is unknown whether multiple beams would be required from a smartphone in $5 \mathrm{G}$, but the possibility that the phone may be simultaneously connected to several devices (Internet of Things, Device-to-Device) other than the base station, favors the use of DMBAs that generate multiple beams, each oriented in a different direction. Digitally controllable arrays offer tremendous flexibility, ease of operation, and programmability.

The transitions $C_{0} \rightarrow C_{2}$ and $C_{1} \rightarrow C_{3}$ are needed to support graceful degradation in the smartphone's operation with respect to array processing. Instead of offering ULA features till the battery completely drains out, it is better to switch to the MRA configuration when the phone goes into the battery-saving (low-power) mode. As mentioned earlier, a $56 \%$ saving is achieved in terms of the feed network, data converters, and power consumption compared to the ULA configuration. Also, the mutual coupling decreases when the ULA is converted to the MRA. The reverse transition $C_{2} \rightarrow C_{0}$ happens when the phone comes back to the normal mode of operation or when it regains the battery charge.

In an equivalent way, when the phone is bent or wrist-wrapped, it will be in the $C_{1}$ state. Under low battery conditions, the bent ULA can be converted into the bent MRA to conserve the resources by realizing the transition $C_{1} \rightarrow C_{3}$. The reverse transition $C_{3} \rightarrow C_{1}$ occurs if the battery regains charge in the bent mode. On the other hand, if the phone is removed from the wrist, flattened, and connected to a power source, then the transitions $C_{3} \rightarrow C_{2} \rightarrow C_{0}$ take place in sequence and the phone comes back to the flat ULA mode.

5.2. The Flexible 5G Smartphone and Its Many Array Processing Modes. The array configurations $C_{0}-C_{3}$ discussed above refer to the ULA that is placed along the $y$-axis. An array of this kind covers only the elevation plane. Hence, another array has to be placed along the $x$-axis to cover the azimuth. Since there is less space along the $x$-axis, a ULA with 8-elements can be considered. However, we assume this array to be fixed along the $x$-axis. That is, the phone, and hence the array cannot be flexed in the $x z$-plane. Since there is only one possible configuration or state for the $x$-array, the combined states of both the arrays depend only on the $y$-array and are equivalent to $\mathrm{C}_{0}-\mathrm{C}_{3}$.

As a further extension, the $x$-array could also be allowed to operate as an MRA using the element combinations

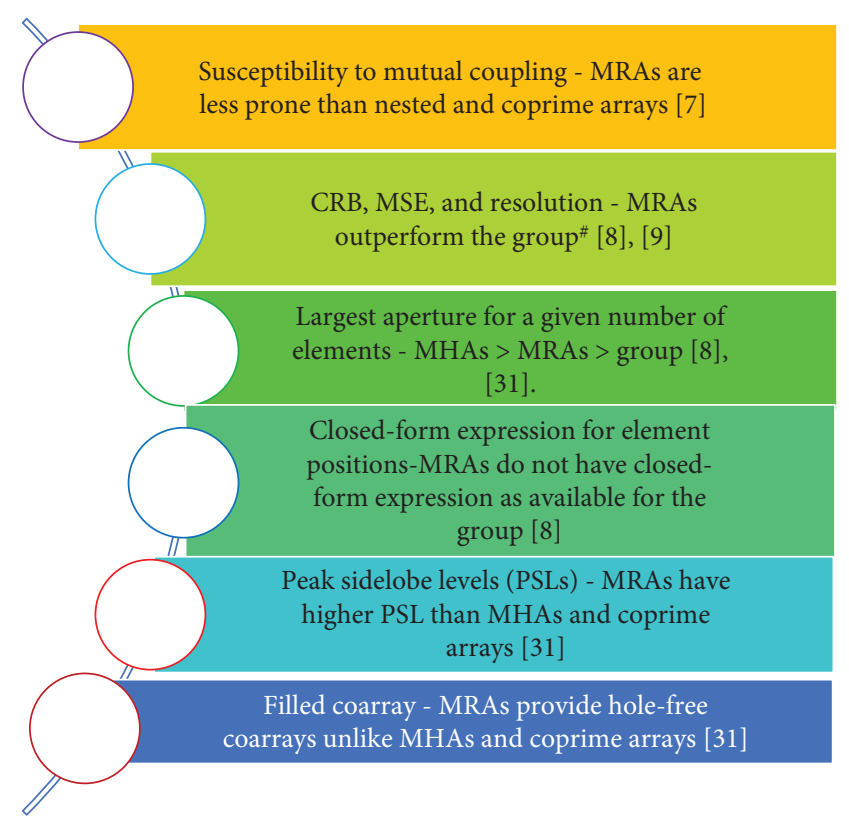

FIgURe 17: Properties of MRAs and other sparse arrays. \#The word "group" is used for conciseness in this figure and collectively refers to coprime, nested, and super-nested arrays.

$\{0,1,3,5,7\}$ or $\{0,2,3,6,7\}$ that provide the required aperture $\left(N_{a}=7\right)$ expected of an 8-element ULA. The $x$-array will then have two states of operation, i.e., either as a ULA or as an MRA. Together, the $x$ - and the $y$-arrays would have eight states. The transitions could be derived to understand the interactions between the states. However, we prefer to end the analysis at this point.

\section{Discussion: Benefits of MRAs and Their Suitability for 5 G Systems}

In this section, we highlight the properties of MRAs that make them stand out among linear sparse arrays and show their suitability for $5 \mathrm{G}$ communication. Figure 17 provides a comparison among linear sparse arrays based on criteria such as susceptibility to mutual coupling, ability to provide better resolution, hole-free coarrays, and sidelobe levels.

Minimum hole arrays (MHAs) can provide a larger aperture than MRAs for the same number of array elements and have lower peak sidelobes (PSLs) than MRAs. Coprime arrays processed in the coarray domain also have lower PSLs than MRAs but need more sensor elements (than MRAs and MHAs) to realize the same aperture. MHAs and coprime arrays have holes in the coarray which makes them less efficient than MRAs for estimating spatial correlation [31]. On the other hand, MRAs provide a filled coarray and are suitable for direction-of-arrival (DOA) estimation and beamforming in the coarray domain [32]. Additionally, MRAs possess better properties than coprime, nested, and super-nested arrays in terms of the aperture provided and the angular resolution achieved as evident from Figure 17. 
It was shown in [4] using adaptive combiners and in [5] using adaptive beamformers, that MRAs fare better than ULAs containing the same number of array elements, when the interfering signals are at close angular separations with respect to the desired signal, or when the multipath components have low angular spread (considering a single source, no interferers, and the two-ray multipath model). Interferers or multipath components which lie within the main lobe of the 4-element ULA are easily rejected by the 4-element MRA since it has a narrower main beam (an aperture of $7 d$ ) than the 4-element ULA. Reading between the lines, both the above advantages (close interferers or multipaths with low angular spread) exploit the narrow main beam characteristics of the MRA. Though not undertaken in [5], even if MRAs and ULAs with the same aperture are considered (i.e., a 4element MRA and a 7-element ULA) and the scan angle or the look direction is limited to the main beam, MRAs are advantageous over ULAs as they provide tremendous savings in the number of elements.

Given the sparsity of the millimeter wave (mmWave) channel, it is an advantage in $5 \mathrm{G}$ systems that MRAs perform well under low multipath conditions. The mmWave channel has sparse multipaths (only a few multipath components) and low angular spreads, owing to the small number of significant scatterers [33].

At this point, we would like to state another reason to support the transitions $C_{0} \leftrightarrow C_{2}$ and $C_{1} \leftrightarrow C_{3}$. Low-battery conditions need not be the sole reason for these transitions. The base station, or for that matter, the 5G Wi-Fi access point which is serving the smartphone might sense any of the following conditions:

(i) a good signal-to-noise ratio (SNR)

(ii) no or very few interferers outside the main lobe

(iii) line-of-sight (LOS) path or sparse multipath

In either case, the base station could instruct the smartphone to switch from the ULA mode to the MRA mode to save the resources. MRAs have acceptable performance in all the three conditions. When either the SNR decreases, or the number of interferers increase, or the LOS path is lost, the base station could command the smartphone to resume operation in the ULA mode. The same reason is valid for the transitions $C_{1} \leftrightarrow C_{3}$.

Additionally, the transitions $C_{0} \rightarrow C_{2}$ and $C_{1} \rightarrow C_{3}$ also fit into the framework of green communications enabled by the underlying green signal processing techniques (lesser number of elements imply lower complexity in computations and data conversion). Being able to operate the smartphone in the MRA mode for prolonged durations provides a significant reduction in the overall computational and power requirements of the device. At higher frequencies up in the millimeter band (e.g., $60 \mathrm{GHz}$ ), ULAs and MRAs that provide larger apertures could be considered. For example, a 36-element ULA and an MRA of 10-elements with the same aperture would mean an unbelievable saving of $72 \%$ $(((36-10) / 36) \times 100 \%)$ in terms of the resources.
The advantages offered by MRAs along with their satisfactory operation in bent positions (of course, with proper phase compensation) make them attractive for $5 \mathrm{G}$ applications. In view of the numerous advantages, the two disadvantages, i.e., high PSLs and nonavailability of closedform expressions for element positions, could be easily overlooked. Nevertheless, alongside the design of flexible displays, printed circuit boards, and batteries, the design of flexible arrays would play a key role in making fully flexible phones a practical reality.

\section{Conclusions and Future Scope}

Different array processing modes have been proposed for the antenna array in a 5G smartphone handset. The array could be made to operate either as a ULA or as an MRA. In either case, if the phone is bent into a semicircular arc, it is shown that the array pattern could be restored through phase compensation. The array offers tremendous savings in the resources when it operates in the MRA configuration.

The proposed work presents a lot of scope for future extensions as listed below

(i) Full wave synthesis could be carried out using software packages followed by prototype fabrication and measurements.

(ii) If $2 \mathrm{D}$ arrays are proposed for use in smartphones, it would be useful to study whether phase compensation could recover the pattern of 2D sparse arrays (such as hour-glass arrays [28] and thermos arrays [34]) bent over semicircular surfaces.

(iii) The practical effects of the millimeter wave channel, polarization mismatch, hand blockage, placement of the arrays in the handset, and so on, studied in $[25,35,36]$, could be redone for the case of MRAs and flexible arrays.

\section{Data Availability}

The MATLAB codes used to support the findings of this study are available from the corresponding author upon request.

\section{Conflicts of Interest}

The authors declare that there are no conflicts of interest.

\section{References}

[1] A. Moffet, "Minimum-redundancy linear arrays," IEEE Transactions on Antennas and Propagation, vol. 16, no. 2, pp. 172-175, 1968.

[2] M. Ishiguro, "Minimum redundancy linear arrays for a large number of antennas," Radio Science, vol. 15, no. 6, pp. $1163-$ 1170, 1980.

[3] D. Pearson, S. U. Pillai, and Y. Lee, "An algorithm for nearoptimal placement of sensor elements," IEEE Transactions on Information Theory, vol. 36, no. 6, pp. 1280-1284, 1990. 
[4] M. B. Jorgenson, M. Fattouche, and O. T. Nichols, "An adaptive minimum redundancy array for digital communications," Canadian Journal of Electrical and Computer Engineering, vol. 16, no. 3, pp. 105-111, 1991.

[5] M. Fattouche, M. B. Jorgenson, and S. T. Nichols, "Applications of minimum redundancy arrays in adaptive beamforming," IEE Proceedings H Microwaves, Antennas and Propagation, vol. 138, no. 5, p. 441, 1991.

[6] L. C. Godara and D. A. Gray, "An algorithm for adaptive augmented array beamforming," The Journal of the Acoustical Society of America, vol. 83, no. 6, pp. 2261-2265, 1988.

[7] E. BouDaher, F. Ahmad, M. G. Amin, and A. Hoorfar, "Mutual coupling effect and compensation in non-uniform arrays for direction-of-arrival estimation," Digital Signal Processing, vol. 61, pp. 3-14, 2017.

[8] C.-L. Liu and P. P. Vaidyanathan, "Cramér-Rao bounds for coprime and other sparse arrays, which find more sources than sensors," Digital Signal Processing, vol. 61, pp. 43-61, 2017.

[9] M. Wang and A. Nehorai, "Coarrays, MUSIC, and the Cramér-Rao bound," IEEE Transactions on Signal Processing, vol. 65, no. 4, pp. 933-946, 2017.

[10] R. A. Monzingo, R. L. Haupt, and T. W. Miller, Introduction to Adaptive Arrays, N. C. Raleigh, Ed., SciTech Pub., 2nd edition, 2011, http://digital-library.theiet.org/content/ books/ew/sbew046e.

[11] V. Semkin, A. Bisognin, M. Kyrö et al., "Conformal antenna array for millimeter-wave communications: performance evaluation," International Journal of Microwave and Wireless Technologies, vol. 9, no. 1, pp. 241-247, 2017.

[12] V. Semkin, F. Ferrero, A. Bisognin et al., "Beam switching conformal antenna array for mm-wave communications," IEEE Antennas and Wireless Propagation Letters, vol. 15, p. 1, 2015.

[13] Q. Wang, N. Mu, L. Wang, S. Safavi-Naeini, and J. Liu, "5G MIMO conformal microstrip antenna design," Wireless Communications and Mobile Computing, vol. 2017, Article ID 7616825, 11 pages, 2017.

[14] B. D. Braaten, S. Roy, S. Nariyal et al., "A self-adapting flexible (SELFLEX) antenna array for changing conformal surface applications," IEEE Transactions on Antennas and Propagation, vol. 61, no. 2, pp. 655-665, 2013.

[15] S. Roy, S. Sajal, and B. D. Braaten, "A phase correction technique based on spatial movements of antennas in realtime for designing self-adapting conformal array antennas," Microwave and Optical Technology Letters, vol. 59, no. 12, pp. 3002-3010, 2017.

[16] G. Mansutti, F. Rigobello, S. M. Asif, M. S. Khan, A.-D. Capobianco, and A. Galtarossa, "Main lobe control of a beam tilting antenna array laid on a deformable surface," International Journal of Antennas and Propagation, vol. 2018, Article ID 2521953, 6 pages, 2018.

[17] Q. L. Yang, Y. L. Ban, K. Kang, C. Y. D. Sim, and G. Wu, "SIW multibeam array for 5G mobile devices," IEEE Access, vol. 4, pp. 2788-2796, 2016.

[18] N. Ojaroudiparchin, M. Shen, S. Zhang, and G. F. Pedersen, “A switchable 3-D-coverage-phased array antenna package for 5G mobile terminals," IEEE Antennas and Wireless Propagation Letters, vol. 15, pp. 1747-1750, 2016.

[19] B. Yu, K. Yang, C. Y. D. Sim, and G. Yang, "A novel $28 \mathrm{GHz}$ beam steering array for $5 \mathrm{G}$ mobile device with metallic casing application," IEEE Transactions on Antennas and Propagation, vol. 66, no. 1, pp. 462-466, 2018.

[20] S. Lee, J. M. Lee, K. C. Hwang, S. Park, and S. Lee, "Hybrid robust optimization for the design of a smartphone metal frame antenna," International Journal of Antennas and Propagation, vol. 2018, Article ID 6325806, 8 pages, 2018.

[21] A. C. Sodré, I. F. da Costa, R. A. dos Santos, H. R. D. Filgueiras, and D. H. Spadoti, "Waveguide-based antenna arrays for $5 \mathrm{G}$ networks," International Journal of Antennas and Propagation, vol. 2018, Article ID 5472045, 10 pages, 2018.

[22] A. Camps, A. Cardama, and D. Infantes, "Synthesis of large low-redundancy linear arrays," IEEE Transactions on Antennas and Propagation, vol. 49, no. 12, pp. 1881-1883, 2001.

[23] F. Gross, Smart Antennas with MATLAB, McGraw-Hill Education, 2nd edition, 2015.

[24] H. L. V. Trees, Detection, Estimation, and Modulation Theory, Optimum Array Processing, John Wiley \& Sons, 2004.

[25] W. Hong, K. H. Baek, and S. Ko, "Millimeter-wave 5G antennas for smartphones: overview and experimental demonstration," IEEE Transactions on Antennas and Propagation, vol. 65, no. 12, pp. 6250-6261, 2017.

[26] R. L. Haupt, Antenna Arrays: A Computational Approach, John Wiley \& Sons, Wiley-IEEE press, 2010.

[27] P. S. Naidu, Sensor Array Signal Processing, CRC Press, 2nd edition, 2009, https://www.crcpress.com/Sensor-Array-SignalProcessing-Second-Edition/Naidu/p/book/9781138113978.

[28] M. Haghzadeh, H. M. Jaradat, C. Armiento, and A. Akyurtlu, "Design and simulation of fully printable conformal antennas with BST/polymer composite based phase shifters," Progress In Electromagnetics Research C, vol. 62, pp. 167-178, 2016.

[29] A. Nordrum, "Fold-up screens could make their big debut [top tech 2017],” IEEE Spectrum, vol. 54, no. 1, pp. 50-51, 2017.

[30] W. Hong, Z. H. Jiang, C. Yu et al., "Multibeam antenna technologies for $5 \mathrm{G}$ wireless communications," IEEE Transactions on Antennas and Propagation, vol. 65, no. 12, pp. 6231-6249, 2017.

[31] F. S. Rawnaque and J. R. Buck, "Comparing the effect of aperture extension on the peak sidelobe level of sparse arrays," The Journal of the Acoustical Society of America, vol. 142, no. 5, pp. EL467-EL472, 2017.

[32] C. L. Liu and P. P. Vaidyanathan, "Hourglass arrays and other novel 2-D sparse arrays with reduced mutual coupling," IEEE Transactions on Signal Processing, vol. 65, no. 13, pp. 33693383, 2017.

[33] T. Bai, A. Alkhateeb, and R. Heath, "Coverage and capacity of millimeter-wave cellular networks," IEEE Communications Magazine, vol. 52, no. 9, pp. 70-77, 2014.

[34] L. Sun, M. Yang, and B. Chen, "Thermos Array: Two-Dimensional Sparse Array with Reduced Mutual Coupling," International Journal of Antennas and Propagation, vol. 2018, Article ID 3624514, 8 pages, 2018.

[35] Y. Huo, X. Dong, and W. Xu, "5G cellular user equipment: from theory to practical hardware design," IEEE Access, vol. 5, pp. 13992-14010, 2017.

[36] T. S. Rappaport, Y. Xing, G. R. MacCartney, A. F. Molisch, E. Mellios, and J. Zhang, "Overview of millimeter wave communications for fifth-generation (5G) wireless networkswith a focus on propagation models," IEEE Transactions on Antennas and Propagation, vol. 65, no. 12, pp. 6213-6230, 2017. 


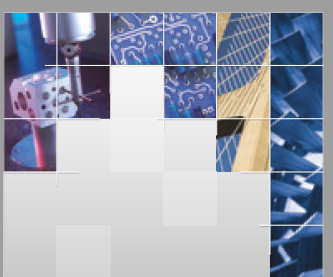

\section{Enfincering}
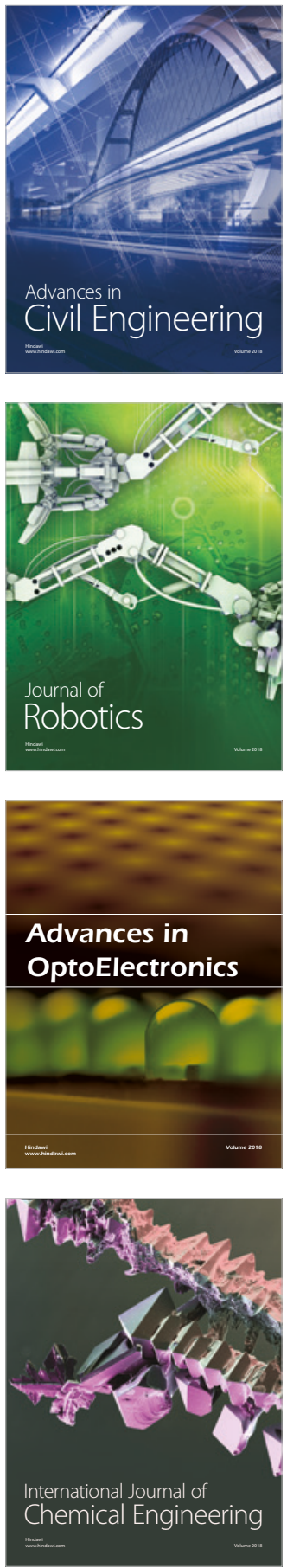

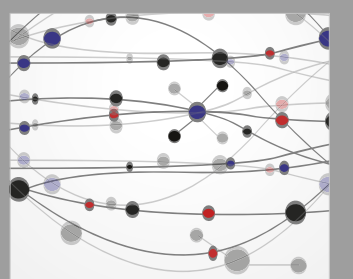

\section{Rotating \\ Machinery}

The Scientific World Journal

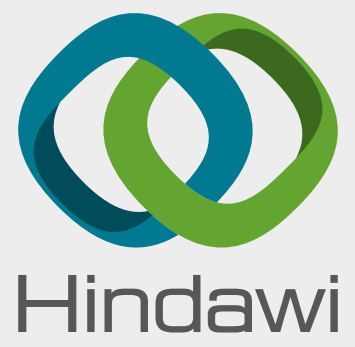

Submit your manuscripts at

www.hindawi.com
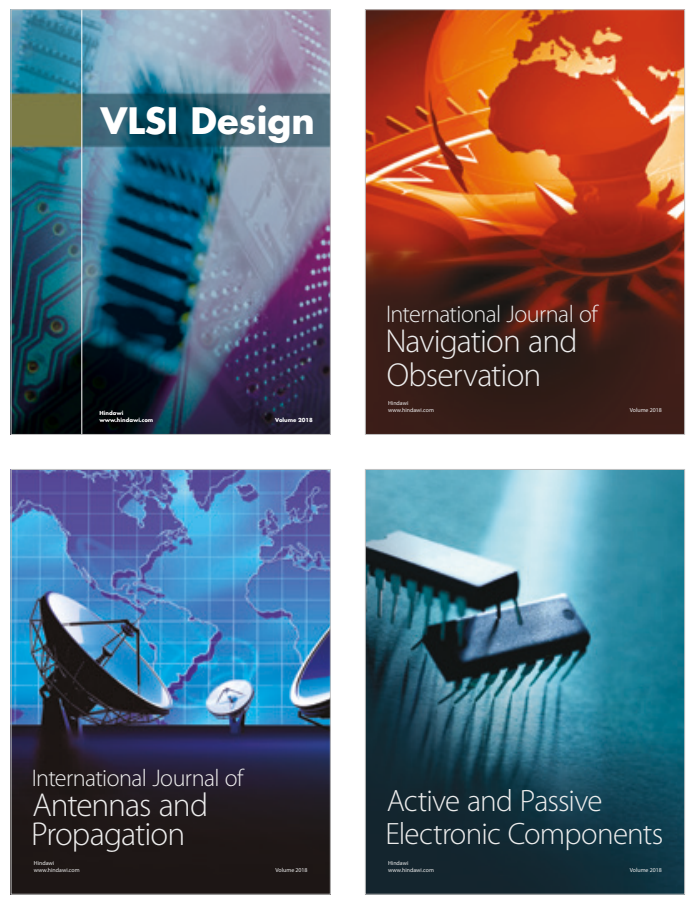
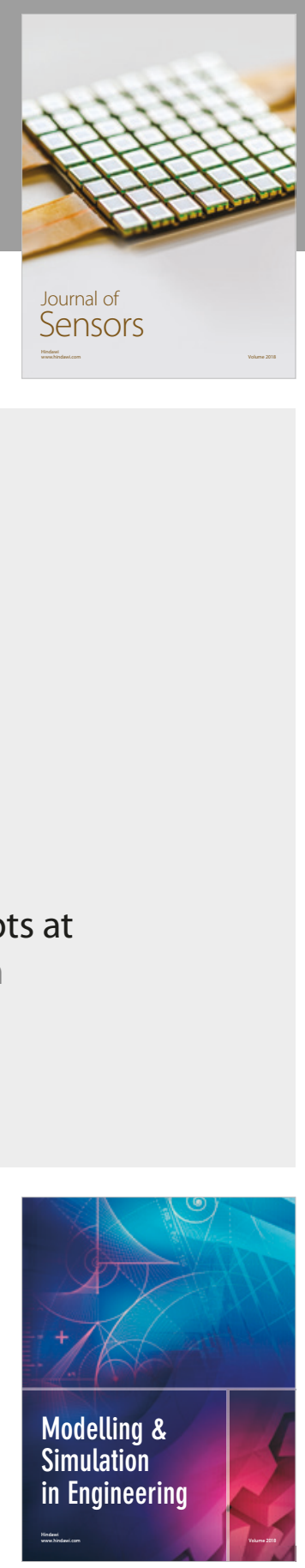

\section{Advances \\ Multimedia}
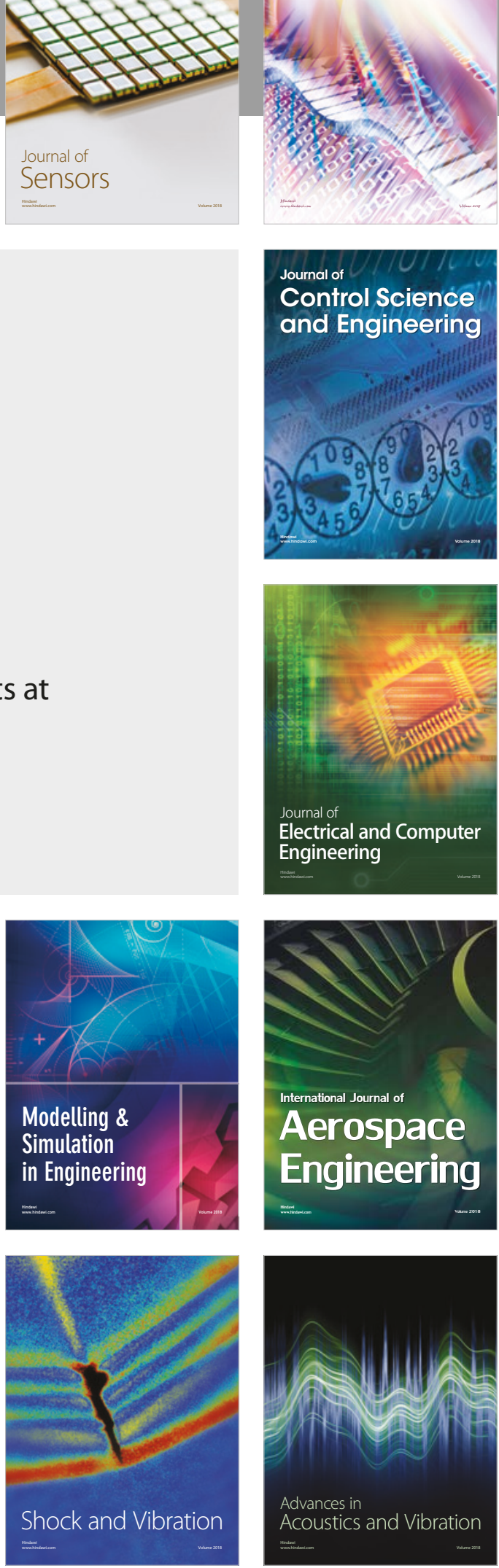